\title{
Systematic Review on Therapeutic Applications of Yeast 'Saccharomyces'
}

\section{Olivia Mondal ${ }^{1}$, Devanshi Khanna ${ }^{2}$, Shalja Panwar ${ }^{3}$, Shilpa Negi ${ }^{4}$, Sneha Basu ${ }^{5}$}

${ }^{1}$ School of Applied and Interdisciplinary Sciences, Indian Association for the Cultivation of Sciences, Jadavpur, Kolkata, India

${ }^{2}$ Clinical Research Department, School of Biosciences (SBS), Apeejay Stya University, Palwal- Sohna Road, Gurugram, India

${ }^{3}$ Centre of Excellence Laboratory, Department of Biochemistry and Plant Physiology, College of Biotechnology, Sardar Vallabhbhai Patel University of Agriculture \& Technology, Meerut, India

${ }^{4}$ Department of Zoology, HNB Garhwal University, Uttarakhand, India

${ }^{5}$ Department of Biotechnology, SRM University, SRM Nagar, Kattankulathur, India

\begin{tabular}{|c|c|}
\hline & ABSTRACT \\
\hline $\begin{array}{l}\text { Article Info } \\
\text { Volume } 8 \text {, Issue } 2 \\
\text { Page Number : 174-197 }\end{array}$ & $\begin{array}{l}\text { This article is an examination of the therapeutic applications of yeast } \\
\text { 'Saccharomyces'. The scientific development and subsequent usage of } \\
\text { Saccharomyces as a potential biodrug continue to influence researchers } \\
\text { worldwide today. This article examines the research done and published by } \\
\text { researchers and scientists. Consideration of current trends and data in scientific } \\
\text { queries and demonstrates further aspects of therapeutic applications of yeast } \\
\text { 'Saccharomyces. Additionally, this article explores options for the treatment of } \\
\text { various ailments by using Saccharomyces as a probiotic. Being a probiotic, }\end{array}$ \\
\hline $\begin{array}{l}\text { Publication Issue } \\
\text { March-April-2021 }\end{array}$ & $\begin{array}{l}\text { Saccharomyces has antimicrobial activity, can modulate immune responses and } \\
\text { competes with pathogens for adhesion sites and nutrients. It showed efficient } \\
\text { results and can be used in diarrhea, acute and chronic gastrointestinal diseases, } \\
\text { Acute necrotizing pancreatitis (ANP), Giardiasis, Autoimmune } \\
\text { Encephalomyelitis (EAE), Crohn's disease, etc. It is also proved to reduced the } \\
\text { vaginal bacterial and fungal loads, thereby aiding the treatment of vaginal }\end{array}$ \\
\hline Article History & diseases; even oral administration has been tested to show positive results. \\
\hline Accepted : 10 March 2021 & Keywords : Probiotic, Recombinant Yeast, Intestinal disorders, \\
\hline Published : 18 March 2021 & Immunomodulation, Vaginal Infections \\
\hline
\end{tabular}

\section{INTRODUCTION}

A genus of fungi is Saccharomyces which includes many species of yeast. This study primarily focuses on Saccharomyces boulardii and Saccharomyces cerevisiae. Saccharomyces boulardii is the first strain that was studied for use as probiotics in human medicine. Saccharomyces cerevisiae and
Saccharomyces boulardii, when used as a probiotic feed additive, inhibits toxins, improves microflora, has antimicrobial activity, can modulate immune responses and competes with pathogens for adhesion sites and nutrient. The use of probiotics in therapy had various advantages, including low cost of treatment, few side-effects and drug resistance. It also leads to the restoration of bacterial imbalances. 
The average estimated incidence of diarrhea in children aged 0-6 years was 1.71 and 1.09 episodes/person/year in rural and urban areas. In India, presently the diarrhoeal morbidity stands at 1.07 million cases, and mortality stands at 2040 in these children. In India, prevalence rates of Giardia infection in patients with diarrhea range from $0.4 \%$ to $70 \%$, and asymptomatic cyst passage has been found to be as high as $50 \%$ in rural southern India. This being the statistics of just two of many diseases that can be treated by Saccharomyces, one can well imagine the importance of better treatment, hence proving its importance. There have been several past attempts in treating these conditions with antimicrobial and other forms of therapy. But the most advantageous point of yeast is that it is not affected by antibacterial drugs and thus can act in synergism with them. Later it can be treated with an antifungal drug if needed. High mucus production in gerbils infected with Giardia was observed in patients administered with Saccharomyces boulardii, which showed a reduction in parasitic load by inhibiting the adherence of trophozoite on intestinal epithelium. Thereafter the oxidative stress produced due to the infection was neutralized, which together prevented Giardiasis.

Saccharomyces cerevisiae displayed slight/moderate antioxidant and anticancer properties. The administration of Saccharomyces boulardii caused a reduction in hemorrhage and inflammatory infiltration, necrosis of hepatocytes caused due to various conditions like autoimmune diseases, druginduced liver injury (DILI), acute viral infections from hepatitis and ALF(acute liver failure). Saccharomyces is now used for the expression of heterologous proteins with therapeutic value against infectious diseases along with recombinant yeast (WRY), yeast surface display (YSD) and virus-like particles (VLPs), a strategy in the fight against infectious diseases and cancer with the present status of yeast-based vaccines in clinical trials.
Saccharomyces is considered an ideal system for the expression and accumulation of high levels of recombinant interfering RNA. Saccharomyces is also engineered to express antigens specific to or associated with tumor cells like Ras gene mutation, or carcinoembryonic antigen had been shown to act as a potential therapeutic vaccine. In the near future, yeast-based edible or oral vaccines may be expected on shelves in medical dispensaries or clinical stores.

Crohn's disease is believed to destroy the intestinal microbiota, and regeneration takes a long time, during which chemical relapses are very common. Saccharomyces boulardii is very similar to Saccharomyces cerevisiae due to which on the administration of this strain, the same kind of antibodies is produced in the host system, which is very specific against Crohn's disease. Saccharomyces cerevisiae is potent in decreasing pro-inflammatory cytokines and increasing $\mathrm{T}$ cells and other immune cells of innate and adaptive immunity. Saccharomyces boulardii and Saccharomyces cerevisiae are identical genetically but differ in resistance to temperature, acidic stressors and growth characteristics were effective in reversing most but not all of the stress induced dysmotility. They have shown autoaggregation ability, cell surface hydrophobicity, antimicrobial activity against bacteria and fungi, strong antioxidant activity, reducing power, nitric oxide and hydroxyl radical scavenging activity, brine shrimp cytotoxicity, acute toxicity, metal ion chelating activity, antibacterial activity and are found to be tolerant to stresses and produce vitamin, enzymes that prevail inside the human body.

\section{METHODS AND MATERIAL}

This study was conducted using four databases: Google Scholar, SAGE, DOAJ and PubMed. The selection of papers was made based on keywords and themes relevant to this review. Further, the published 
papers from these databases were arranged in systemic order with respect to the year of publication.

\section{RESULTS AND DISCUSSION}

\subsection{Saccharomyces boulardii ${ }^{1-10}$}

\section{Saccharomyces boulardii as a therapeutic agent for} Pseudomembranous Colitis

Clostridium difficile, a pathogen that causes Pseudomembranous colitis (PMC), produces two cytotoxins; Cytotoxin A that causes increased fluid secretion into the colonic lumen by altering the cytoskeleton and tight junctions between mucosal epithelial cells and Cytotoxin B causes electrophysiological changes and tissue damage leads to release of inflammation mediators by mucosal cells and immune system cellular response. The standard therapy available for Clostridium difficile- positive diarrhea and PMC like Vancomycin and Metronidazole is not specific to one organism and causes the disturbance of normal flora, while Saccharomyces boulardii does not disturb the normal flora and can be administered orally. The study investigates the effect of Saccharomyces boulardii on the prevention of Pseudomembranous colitis.

The protection of Saccharomyces boulardii against PMC was measured with the help of three mechanisms. There was a decrease in the activity of enzymes such as lactase, maltase, sucrase in PMC, which can lead to diarrhea. After the administration of Saccharomyces boulardii, an increase in the activity of these enzymes was noted and was statistically significant compared with baseline. The second mechanism involved measuring the impact through secretory antibody immunoglobulin $\alpha \operatorname{IgA}$. IgA inhibits the proliferation of Clostridium difficile by preventing its attachment to the intestinal wall and therefore prevents the production of toxins. The third mechanism involved investigating the effect of protease produced by Saccharomyces boulardii on cytotoxins A and B. In addition to monotherapy, the effect of this probiotic yeast was also observed in combinations.

It was concluded that no adverse effects (other than thirst and constipation) were reported in treatment with Saccharomyces boulardii. Administered as an oral therapy, it can prevent the development of diarrhea and PMC. In combination studies with Vancomycin and Metronidazole (administered to patients with active-antibody-associated diarrhea), Saccharomyces boulardii did not prevent the first relapse. However, it reduced second or further relapse to PMC.

\section{Impact of Saccharomyces boulardii on diarrheal pathogens}

Diarrhea affects the bowel's complex ecosystem and causes the invading pathogens to colonize it. Living microorganisms can be used for the prevention and treatment of intestinal infections. Probiotics or the biotherapeutic agents like Lactobacilli, Bifidobacterium and Saccharomyces boulardii can be used for gastrointestinal infections. Steady-state concentrations of Saccharomyces boulardii were noted in the colon within three days in pharmacokinetic studies, which was removed 2-5 days from the stool after administration. Saccharomyces boulardii has a protective effect against enteric pathogens. Being a yeast, it shows resistance to antibacterial antibiotics. Therefore it is better than bacterial biotherapeutic agents. However, antifungal agents such as nystatin were able to remove it from the digestive tract.

Table 1 Effects of Saccharomyces boulardii on different diarrheal pathogens

\begin{tabular}{|l|l|}
\hline PATHOGEN & EFFECT OF S. boulardii \\
\hline Clostridium difficile & Toxin A and B, associated \\
& with Clostridium difficile, \\
& were directly affected by \\
& Saccharomyces boulardii. \\
& Yeast's effects of \\
& Saccharomyces boulardii \\
\hline
\end{tabular}




\begin{tabular}{|l|l|}
\hline & $\begin{array}{l}\text { serine protease against } \\
\text { Clostridium difficile were } \\
\text { noted partially. }\end{array}$ \\
\hline Vibrio cholerae & $\begin{array}{l}\text { It was observed that a } \\
\text { substance produced by } \\
\text { Saccharomyces boulardii } \\
\text { interfere with Ca- } \\
\text { mediated chloride } \\
\text { secretion and resulted in } \\
\text { antidiarrheal effects. }\end{array}$ \\
\hline Enterobacteriaceae & $\begin{array}{l}\text { Phosphorylation of } \\
\text { ERK1/2 mitogen-activated } \\
\text { protein kinase was } \\
\text { attenuated by } \\
\text { Sacharomyces boulardii; } \\
\text { the modulation of the } \\
\text { ERK1/2 pathway resulted } \\
\text { in the decrease in bacteria. }\end{array}$ \\
\hline
\end{tabular}

The mechanism of action of Saccharomyces boulardii in different gastrointestinal pathogens was evaluated. The antimicrobial substances produced as a $54-\mathrm{kDa}$ protease and $120-\mathrm{kDa}$ protein inhibited Clostridium difficile and Vibrio cholerae infections. An antagonist effect against EPEC infection was exerted by Saccharomyces boulardii that led to the preservation of barrier function in infected cells; the signal transduction pathway during EPEC infection was also modulated by this yeast. It protected the host against diarrheal pathogens and infection by increasing the host's intestinal mucosal immune response. Therefore, Saccharomyces boulardii can be used as a therapeutic agent in Clostridium difficile-associated disease and in the prevention of diarrheal infectious pathogens.

Effects of Saccharomyces boulardii in combination with antibiotics in acute amoebiasis

Amoebiasis is caused by entamoeba histolytica, an organism that feeds on intestinal contents and can also cause abscess when moved from bowel to liver and other organs. Although this organism is avirulent, it can become virulent in people with malnutrition, immune suppression, alterations in the intestinal flora.
Additionally, it exists as cysts in the intestine. Cysts can be treated with the help of luminal amoebicides like iodoquinol, paromomycin or diloxanide furoate. Saccharomyces boulardii, a saprophytic and thermophilic yeast, is resistant to gastric juice and can grow in the gastrointestinal tract. The use of Saccharomyces boulardii in addition to the standard treatment for invasive amoebiasis was being assessed in this study. It involved the study of the efficacy of Saccharomyces boulardii with antibiotics in amoebiasis.

The study involved 57 participants, out of which 2 participants were excluded due to non-compliance. It was observed that the mean duration of diarrhea, abdominal pain and fever was reduced to half while there was no effect on headache.

Saccharomyces boulardii was effective for the treatment and prevention of septic enteritis. Additionally, it proved to be helpful in traveler's diarrhea, colitis and Crohn's disease]. It inhibited the enteropathogens and showed antidiarrheal property; it can be used for diarrhea in children. In healthy volunteers, it also showed the tropical effects on the small intestine. Administration of lyophilized Saccharomyces boulardii with antibiotics resulted in the reduction of cysts and symptoms, which may be due to the restoration of normal gut flora. Thus, it proved to be useful in addition to the standard treatment for the treatment of amoebic dysentery.

Table 2 Actions of Saccharomyces boulardii

\begin{tabular}{|l|l|}
\hline ANTIMICROBIAL & $\begin{array}{l}\text { Inhibits bacteria and } \\
\text { ACTIVITY }\end{array}$ \\
$\begin{array}{l}\text { neutralizes bacterial } \\
\text { virulence factors; reduces } \\
\text { gut translocation of } \\
\text { pathogens }\end{array}$ \\
\hline $\begin{array}{l}\text { ANTITOXIN } \\
\text { EFFECTS }\end{array}$ & $\begin{array}{l}\text { Inhibits toxin receptor } \\
\text { binding sites; causes the } \\
\text { secretion of enzymatic } \\
\text { proteins }\end{array}$ \\
\hline $\begin{array}{l}\text { IMMUNE } \\
\text { RESPONSES }\end{array}$ & $\begin{array}{l}\text { Have effects in innate and } \\
\text { adaptive immunity; promote }\end{array}$ \\
\hline
\end{tabular}




\begin{tabular}{|l|l|}
\hline & $\begin{array}{l}\text { mucosal anti-inflammatory } \\
\text { signaling effects; reduces } \\
\text { pro-inflammatory responses. }\end{array}$ \\
\hline $\begin{array}{l}\text { EFFECTS ON } \\
\text { INTESTINAL }\end{array}$ & $\begin{array}{l}\text { It prevents apoptosis, } \\
\text { reduces mucositis, causes } \\
\text { MUCOSA }\end{array}$ \\
& $\begin{array}{l}\text { amines; restoration of fluid } \\
\text { transport pathways. }\end{array}$ \\
\hline
\end{tabular}

Table 3 Effect of Saccharomyces boulardii

\begin{tabular}{|l|l|}
\hline ACUTE GASTROINTESTINAL DISEASES \\
\hline $\begin{array}{l}\text { Antibiotic- } \\
\text { associated Diarrhea }\end{array}$ & Efficacious in preventing AAD \\
\hline $\begin{array}{l}\text { Clostridium } \\
\text { difficile Infection }\end{array}$ & $\begin{array}{l}\text { No data obtained compelled the } \\
\text { routine use of Saccharomyces } \\
\text { boulardii for the treatment of } \\
\text { CDI, although small data in } \\
\text { children stated the effect of } \\
\text { Saccharomyces boulardii in } \\
\text { CDI. }\end{array}$ \\
\hline Acute Diarrhea & $\begin{array}{l}\text { It May be effective as an } \\
\text { adjunctive therapy. }\end{array}$ \\
\hline Persistent Diarrhea & Useful in PD \\
\hline $\begin{array}{l}\text { Enteral nutrition- } \\
\text { related Diarrhea }\end{array}$ & $\begin{array}{l}\text { The reduction was noted in } \\
\text { Saccharomyces boulardii group. }\end{array}$ \\
\hline Traveler's Diarrhea & $\begin{array}{l}\text { Saccharomyces boulardii was } \\
\text { effective. }\end{array}$ \\
\hline $\begin{array}{l}\text { Helicobacter pylori } \\
\text { infection }\end{array}$ & $\begin{array}{l}\text { It was effective in reducing the } \\
\text { side-effects of standard triple } \\
\text { therapy, although it was not } \\
\text { able to eradicate it. }\end{array}$ \\
\hline CHRONIC GASTROINTESTINAL DISEASES \\
\hline Crohn's Disease & $\begin{array}{l}\text { Relapsed cases were less, and } \\
\text { improved results compared to } \\
\text { placebo were noted. }\end{array}$ \\
\hline diarrhea & $\begin{array}{l}\text { Reduce and maintain remission } \\
\text { of Ulcerative colitis }\end{array}$ \\
\hline Ulcerative Colitis
\end{tabular}

Probiotics are used in the treatment and prevention of gastrointestinal diseases and systemic inflammatory processes. Cases of fungemia after the administration of probiotics in immunocompromised patients have been reported. A case of Saccharomyces cerevisiae var. boulardii fungemia in an immunocompromised patient was noted after the treatment with probiotic of Saccharomyces cerevisiae var. boulardii. The use of probiotics has caused more cases of fungemia. However, clinical trials are not successful in establishing the positive effects of probiotic use. An immunocompromised patient developed diarrhea after series of treatments for frontal lobe glioblastoma multiforme. The patient was prescribed lyophilized Saccharomyces boulardii; stool analysis showed the presence of fecal leukocytes and Clostridium difficile A and B toxins, after which Metronidazole was prescribed. The culture revealed the presence of Saccharomyces cerevisiae var. Boulardii fungemia was detected, after which probiotic treatment was discontinued. The use of Saccharomyces cerevisiae var. boulardii was established to exert an effect against Clostridium difficile by neutralizing toxin A and $\mathrm{B}$ due to the production of proteases. It was being used in antibody-associated diarrhea and CDI treatment. However, as reported in many cases, patients with severe systemic gastrointestinal disease or immuno-suppressed individuals it has reported causing fungemia, specifically from Saccharomyces boulardii. Therefore, caution must be taken, especially in the case of critically ill patients in the use of probiotics for treatment. 
Impact of Saccharomyces boulardii CNCM I-745 on microbiota-gut-brain axis in a humanized mouse model of Irritable Bowel Syndrome

Irritable bowel syndrome (IBS), accompanied by psychiatric co-morbidities like depression and anxiety, is a disorder of gut-brain communication. It is associated with chronic abdominal pain, altered bowel habits. Patients with IBS show disturbances in composition, temporal stability, metabolic activity. The study involved the impact of probiotics on gut dysfunction and psychiatric co-morbidities. A strain of Saccharomyces boulardii, CNCM I-745, was used to examine the therapeutic effects of this strain on anxiety-like behavior and gut dysfunction in a humanized mouse model of Irritable Bowel Syndrome with anxiety.

Anxiety-like behavior was normalized due to the administration of the Saccharomyces boulardii. 30\% faster gastrointestinal transit was observed in $\mathrm{Ex}^{-}$ germ-free mice induced with microbiota from IBS+A patient than mice colonized with HC (Healthy Control) microbiota, although the gastrointestinal transit did not cross the statistical significance level. A two-fold increase in the expression Trpv1, the gene associated with visceral hypersensitivity and anxiety, was noted in IBS+A microbiota colonized mice than in the control group. The expression was decreased after the administration of Saccharomyces boulardii, although the result was not statistically significant. The microbiota profiles of IBS+A and HC were seen to be clustered in beta-diversity analysis while the profiles of IBS+A receiving water or Saccharomyces boulardii coincided. The differences discovered during the analysis at family and genus levels between IBS+A and HC were normalized after the treatment with Saccharomyces boulardii. The number of bacterial genes for indole alkaloid biosynthesis, indoles are the activators of Aryl hydrocarbon Receptor (AhR), were 20-fold higher in IBS+A mice treated with Saccharomyces boulardii than in those receiving water. The AhR activity (measured in mouse feces) was increased in IBS+A treated with Saccharomyces boulardii than in control and IBS+A that received water. Additionally, indol-3-acetic acid (IAA) and indole-producer Lactobacillus were also higher in Saccharomyces boulardii treated IBS+A. Therefore, it was noted that microbial indole production was increased by Saccharomyces boulardii in vitro.

Several factors contributed to the development of IBS, like intestinal dysfunction, impaired brain-gut interactions, psychosocial status. Induction of fecal microbiota from IBS+A in germ-free mice resulted in gut dysfunction and anxiety-like behavior. It can be inferred that the anxiety-like behavior was reduced after the treatment of Saccharomyces boulardii in a humanized mouse model through various mechanisms like increased production of indole metabolites, IAA, suggesting the usefulness of this strain of Saccharomyces boulardii in IBS. Therefore, this probiotic agent can be used in patients with Intestinal Bowel Syndrome and for its co-morbidities.

Clinical evidence : as extracted from clinicaltrials.gov Saccharomyces boulardii in Irritable Bowel Syndrome (SB-IBS)

It was an interventional, randomized, parallel assignment, Triple (Participant, Investigator, Outcomes Assessor) masked study. The primary outcome included testing clinical symptoms, cytokine levels, histology features before and after the intervention within six weeks and then the impact of Saccharomyces boulardii in the safety of the drug was assessed. (no results posted)

\section{The Effect of Probiotics on E.coli induced Gastroenteritis}

The study involved 60 participants in an interventional, randomized, triple masked (Participant, Investigator, Outcome assessor) 
experiment. It involved two groups: Probiotic group and Control group. The primary outcome was to compare the enterotoxigenic $E$. coli (ETEC) excretion over time curve between the two groups. Bowel habits, the severity of gastrointestinal symptoms were also measured. (no results posted).

Effects of Saccharomyces boulardii and Amoxicillin/Clavulanate on Gut Microbiota

The trial involved four groups: ProbioticSaccharomyces boulardii; Antibiotic- Amoxicillin Clavulanate; Combination of both; Control. It was a randomized, parallel assignment, open-label trial. The results were assessed according to the Gastrointestinal System Rating Scale; an increased score meant increased symptoms. It was observed that the mean gastrointestinal symptom rating scale scores were lower in the combination group in comparison to other groups (data are taken till 21 days). Also, most of the days, the value was less in the probiotic group than the antibiotic group.

\section{Effectiveness of Saccharomyces boulardii for} Gastrointestinal Bacterial-overgrowth in Systemic Sclerosis

The study was carried out to test the effectiveness of Saccharomyces boulardii oral tablet (given for 2 weeks, then 2 weeks rest and continued for 2 months) in combination with Metronidazole. Randomized, parallel assignment, the open clinical trial consisted of 39 participants. Breath test using hydrogen monitor was used to see small intestinal bacterial overgrowth and gastrointestinal symptoms were noted before and after the intervention. (results not posted).

Impact of Additional Treatment with Saccharomyces boulardii on Quality of Life in Patients with Mild Forms of Ulcerative Colitis and Crohn's Disease

The trial was carried out to study the effect of Saccharomyces boulardii and dietary supplements in ulcerative colitis and Crohn's disease. The study divided patients into two groups: treatment and control and was assessed after 4 weeks of initiation of therapy through a questionnaire. It was a randomized, parallel assignment trial with Quadruple (Participant, Care Provider, Investigator, Outcomes Assessor) masking. (results not posted).

Efficacy and Safety of Saccharomyces boulardii in the Treatment of Small Intestinal Bacterial Overgrowth

Involved in testing the efficacy of Saccharomyces boulardii with low fermentation diet administration. It was a randomized, parallel assignment, an openlabel trial involving 71 participants. The severity of symptom was measured using Irritable Bowel Syndrome Symptom Severity Scale. (no results posted)

\subsection{Saccharomyces cerevisiae ${ }^{11-21}$}

Saccharomyces cerevisiae used to investigate the response to anticancer agents.

The development of new strategies for cancer therapeutics focuses on yeast Saccharomyces cerevisiae as a model system by the use of mutant yeast panels to mimic cancer phenotypes, screen and study tumor features and synthetic lethal interactions. Saccharomyces cerevisiae, the first eukaryote to have its genome fully sequenced, used as a compatible model organism to study cell cycle control, DNA repair, aging, gene expression, autophagy and molecular and cellular pathways of human diseases, including cancer. Identification of the alkylating agent cisplatin and the topoisomerase II poison mitoxantrone, characterization of the doxorubicin and bleomycin resistant profiles introduces a way to mimic a variety of cancer phenotypes in the yeastconserved genetic background. Yeast-based systems have proved to be a useful tool in small molecules screening for HDAC activity. Yeast Rpd3 deacetylase shares a $60 \%$ identity to human class 1 HDAC proteins. Yeast also presents limitations, its cell wall, lack of important human proteins in anticancer research such as tumor suppression, apoptosis and drug metabolism. The use of Saccharomyces cerevisiae represents a faster and cheaper solution to screen anticancer drugs and cytotoxicity, providing an 
easier and manipulating system to mimic the numerous combination of genetic alterations in cancer cells that may be explored using the synthetic lethal approaches. Treatment effects of beneficial microbes on stress-induced dysmotility microbial effects on intestinal propulsive motility and neuromuscular machinery. Saccharomyces boulardii and Saccharomyces cerevisiae are identical genetically but differ in resistance to temperature, acidic stressors and growth characteristics were effective in reversing most but not all of the stress-induced dysmotility. A need to identify the bioactive molecules released by the yeasts Saccharomyces cerevisiae and Saccharomyces boulardii clinical trials further support the therapeutic potential of Saccharomyces yeasts in humans.

\section{Saccharomyces cerevisiae IFST062013 as a potential probiotic for Antimicrobial and antioxidant activities}

This study tells us the probiotic or health-beneficial potential of Saccharomyces cerevisiae IFST 062013 strain which showed auto-aggregation ability, cell surface hydrophobicity, antimicrobial activity against bacteria and fungi, strong antioxidant activity, reducing power, nitric oxide and hydroxyl radical scavenging activity, brine shrimp cytotoxicity, acute toxicity, metal ion chelating activity, antibacterial activity. Thus, the isolate improves lymphocyte proliferation and cytokine production in treated mice considered to be used therapeutically.

A potential probiotic (Saccharomyces cerevisiae IFST 062013) (Accession no- HM134859.1) was isolated from fruit identified and characterized on the basis of morphological and biochemical characteristics and phylogenetic analysis. Saccharomyces cerevisiae has clinically proven to have a beneficial effect on the host by improving its intestinal microbial balance showing moderate autoaggregation ability and cell surface hydrophobicity that provides adhesion of the microorganisms to intestinal epithelial cells of patients and to be tolerant to stresses and produce vitamin, enzymes that prevail inside the human body. The resistance to antibiotics makes it suitable for use in patients. Culture supernatant showed least antimicrobial activity indicating that the antimicrobial compounds showed antagonistic activity against human pathogens, strong antioxidant activity, reducing power, nitric oxide and hydroxyl radical scavenging activity, significant brine shrimp cytotoxicity and acute toxicity and metal ion chelating activity possessing significant antiinflammatory activity in mice. Saccharomyces cerevisiae strain stimulates a T-lymphocyte specific proliferative and thus maintains a balance between Th1 and Th2 type cytokines, which is important for host immunity. It is clear that microflora of the human body can be prevented by the use of appropriate probiotics. Further research should be done to ensure safe and efficient therapeutic applications.

\section{Therapeutic use of Saccharomyces cerevisiae strain (OBS2) from fermented nectar of toddy palm}

15 yeast strains from the nectar of toddy isolated and characterized. Selected antibiotic resistance strain evaluated in vitro for tolerance to gastrointestinal conditions and for their pathogenicity and adherence to intestinal epithelial cells; as a result, the 2 yeast isolates characterized as Pichia kudriavzevii OBS1 (100\%), and Saccharomyces cerevisiae OBS2 (96\%) using BLAST analysis were deposited in the GenBank data library. Saccharomyces cerevisiae OBS2 displayed slight/moderate antioxidant and anticancer properties. Hence, strain OBS2 can be utilized and explored as a potential probiotic for therapeutic applications.

A total of 53 yeast isolates (OBS1-OBS53) were isolated from the nectar of toddy palm.

Fifteen yeast isolates (OBS1-OBS15) were selected culturally and morphologically characterized, based 
on the results of morphological, cultural and antimicrobial drug sensitivity profiles, 8 isolates were selected. Stress tolerance was tested among the 7 yeast isolates; OBS1 and OBS2 were noticed to tolerate extreme conditions of the gastrointestinal tract like temperature, low $\mathrm{pH}$, organic acids and digestive enzymes, i.e., pepsin and amylase in the stomach and trypsin and bile in the upper intestine expressed a strong adhesive ability of more than 40 yeast cells adhering to the monolayer considered as nonpathogens. Both showed 96\% Saccharomyces cerevisiae and $100 \%$ identity with Pichia kudriavzevii, respectively. Accession number KP998094 for Saccharomyces cerevisiae OBS2 and KP998095 for Pichia kudriavzevii, OBS1 were obtained. Further Therapeutic properties were tested where OBS2 resulted in a slight reduction in percentage cell viability of neuroblastoma cell line IMR32 and increased absorbance with increased concentration of autolysate antioxidant property.

Culturally and morphologically characterized yeast isolates were resistant to the majority of the antibacterial drugs. Based on resilience properties to gastrointestinal parameters, antimicrobial agents' sensitivity, antagonistic activity, pathogenicity, cocultural activity, adherence ability to intestinal epithelial cells, 2 yeast isolates. Selected and characterized as Pichia kudriavzevii OBS1 and Saccharomyces cerevisiae OBS2. Two isolates, Saccharomyces cerevisiae OBS2 have a cytotoxic effect on cancer cells and antioxidant activity. Hence, it can be used for human probiotic and therapeutic applications.

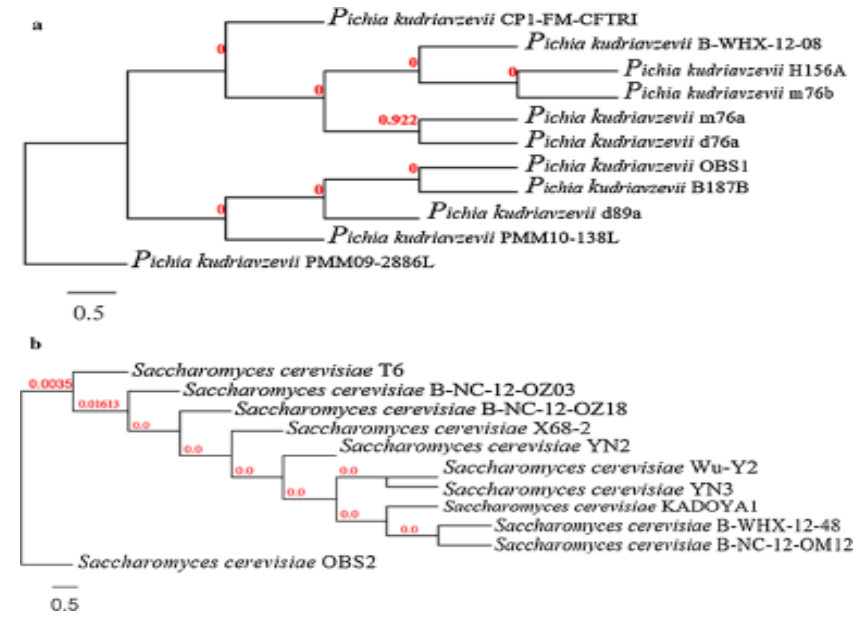

Biotechnological approach and applications of sustainable yeast

Saccharomyces cerevisiae in biotechnology with known genetic and physiological background the availability of large-scale fermentation and biological conversion and in the formation of products such as ethanol, organic acids, amino acids, enzymes, and therapeutic proteins. Thus, sustainable solutions are used to overcome various environmental effects on yeast.

The study mainly focuses on the development of Saccharomyces cerevisiae for the production of chemicals, protein, enzymes. Saccharomyces cerevisiae is the most dominated in cell factories as $40 \%$ of the biopharmaceuticals had been produced by using Chinese Hamster Ovarian cell lines (CHO cells). Saccharomyces cerevisiae is chosen to be the most suited organism as a host in microbial production. Engineered yeast help for the production of fatty acid-derived oleochemicals, biofuels, biochemicals metabolites and enzymes such as invertase and lactase, where Saccharomyces species are used. A lot of yeast strains are involved in the formation of different milk products like cheese. Kluyveromyces lactis and Debaryomyces hansenii strain they have actively grown on soft-cheese model curds (SCMC) surface by taking 31 days periods for the ripening, kafir which proved therapeutic properties as a natural probiotic beverage alcoholic fermented beverages and yogurt. 
Therefore, available tools from yeast engineering, product optimization will be simpler day by day.
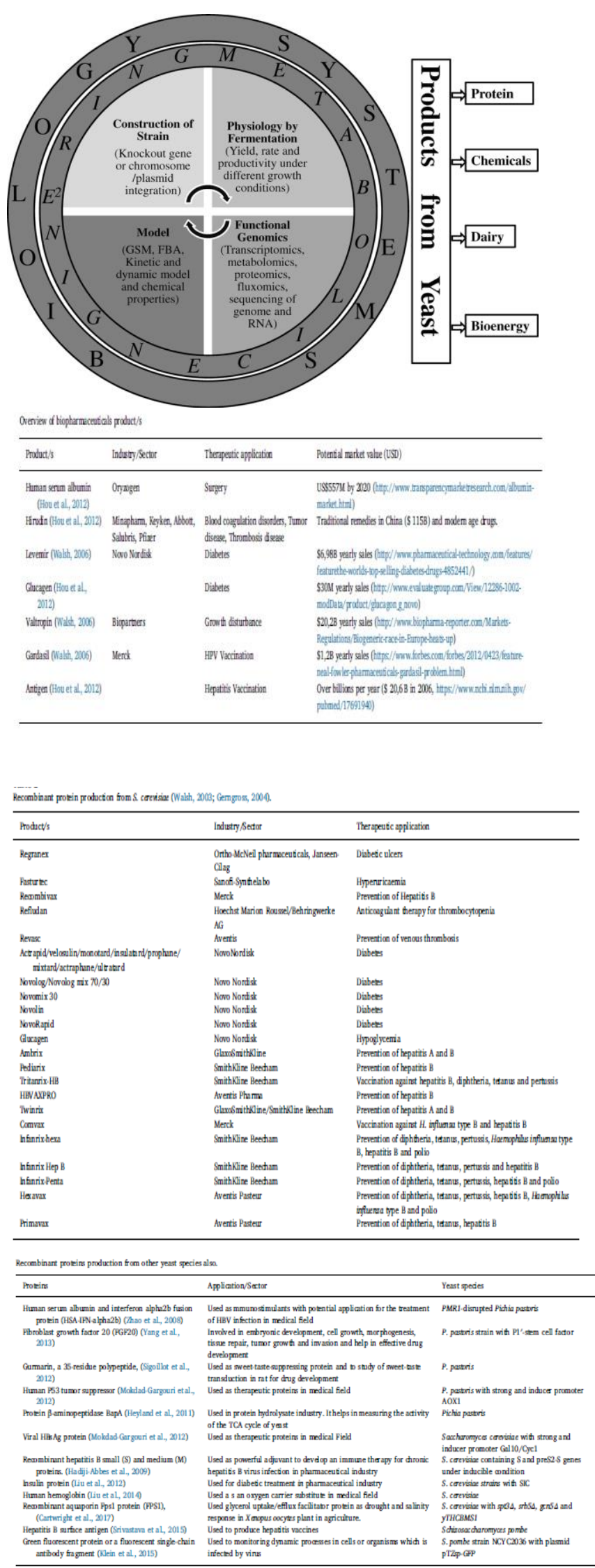

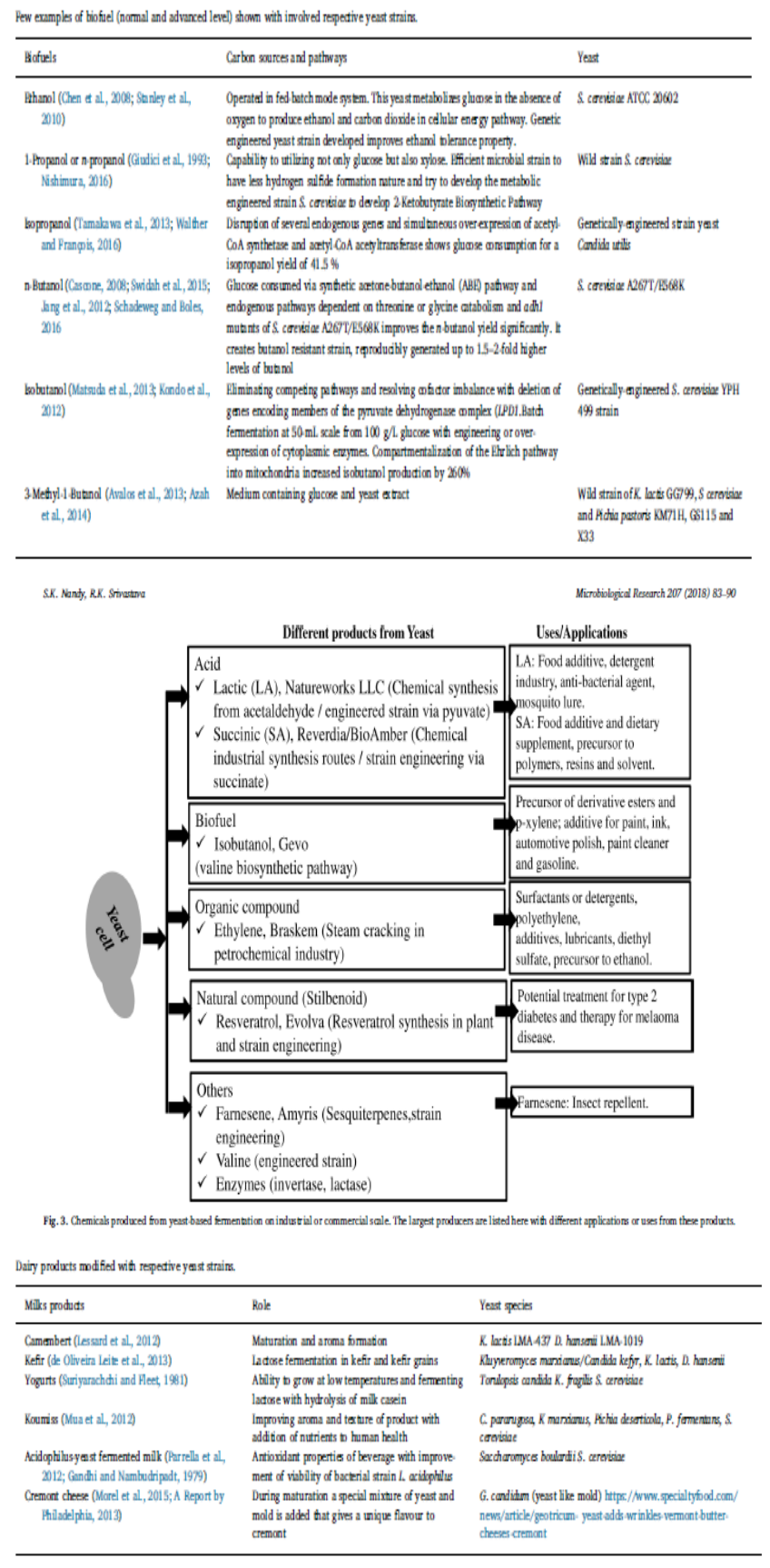

\section{Development and uses of Yeast based vaccines}

This study focuses on Saccharomyces cerevisiae and $P$. pastoris expression of heterologous proteins with therapeutic value against infectious diseases along with recombinant yeast (WRY), yeast surface display (YSD) and virus-like particles (VLPs), a strategy in the fight against infectious diseases and cancer with the present status of yeast-based vaccines in clinical trials. Heterologous protein species, including the first commercialized recombinant vaccine, the hepatitis B 
vaccine, were produced in Saccharomyces cerevisiae. The oral administration of recombinant yeast is important in both the recognition and killing of tumor cells. CEA vaccine in adults with metastatic. DENV envelope (E) protein-based Virus-like particles (VLPs) generated using $P$. pastoris shows results against dengue, HIV, inhibit the growth of $P$. falciparum CC1/HN and 3D7 lines in rabbit and rhesus monkeys, Lactobacillus pleuropneumonia, Aspergillosis and Coccidioidomycosis, Coccidioidomycosis, candidiasis, Necator americanus infection, Ancylostoma caninum and Ancylostomaceylanicum. Boophilus microplus and Ascaris suum. Saccharomyces cerevisiae and $P$. pastoris emerge as a most promising candidate for the expression of heterologous proteins for vaccine development. In the near future, yeast-based edible or oral vaccines may be common on shelves in medical dispensaries or clinical stores.

\section{Saccharomyces cerevisiae a model system for} Interfering RNA Expression and Delivery System

The study is about the commercial development of yeast interfering RNA expression and delivery systems. Saccharomyces cerevisiae is considered an ideal system for the expression and accumulation of high levels of recombinant interfering RNA. Recent research focuses on optimization of interfering RNA expression in Saccharomyces cerevisiae industrialsized scaling of recombinant yeast cultures in which interfering RNA molecules expressed the development of methods for large-scale drying of yeast that preserve interfering RNA integrity and identification of encapsulating agents that promote yeast stability in various environmental conditions. The food and pharmaceutical industry will facilitate further development.

siRNAs can suppress viral infections, tumorigenesis, inflammatory disorders, and cardiovascular disease used for the control of human disease vector insects. Saccharomyces cerevisiae had been investigated as a potential vaccine carrier useful for the oral delivery system for biological molecules to intestinal DC cells. In agricultural biotechnology communities of siRNA induces mosquito larval lethality and also useful in food and alcoholic beverage, bioreactor resulted in higher yields renewable and cost-effective manner. In biopharmaceutical industries, half of the global supply of insulin is produced in Saccharomyces cerevisiae and production of siRNA-based biopharmaceuticals and biopesticides. Therefore, technology is an efficient method for the industrial production of probiotics. Thus, many potential agents for microbial encapsulations are available, and this will be a critical area of exploration for the improvement of yeast RNA delivery systems. The microbe in both the food and pharmaceutical industry will benefit the development of this microbe as a production and delivery system for interfering RNA molecules. Develop scaled methods were used for drying yeast that preserves the integrity of the interfering RNA molecules.

\section{Probiotics based on Saccharomyces cerevisiae as an} antimicrobial agent for the prevention and treatment of vaginal infections

$70 \%$ of women worldwide are affected by the vaginal infection, mainly VVC and BV, which are difficult to treat. Standard antimicrobial agents are used for the prevention and treatment of these vaginal infections. This study focuses on a new approach of yeast-based probiotics VVC and BV are the most prevalent vaginal infections caused by fungi Candida albicans $(C$. albicans) and Trichomonas vaginalis respectively, lead to the destruction of the protective mucus on the vaginal epithelium damaging the female reproductive organs. Various therapeutic approaches Fluconazole amphotericin B, nystatin, and flucytosine for VVC and oral or intravaginal Metronidazole, intravaginal clindamycin cream, clindamycin and tinidazole secnidazole, a second-generation 5-nitroimidazole agent and tinidazole for BV are used with limitation of a long-term defensive barrier. Therefore, probiotics administration represents an alternative 
therapeutical approach for treating vaginal infections. Saccharomyces boulardii CNCM I-745 was the first strain studied. Live and inactivated Saccharomyces cerevisiae and vaginal administration of Saccharomyces proved to reduce the vaginal bacterial and fungal loads. Saccharomyces cerevisiae based approaches open the door to future clinical studies for the treatment of vulvovaginal candidiasis and bacterial vaginosis.

\section{Recombinant Saccharomyces cerevisiae expressing} antigens - Ras mutations and Carcinoembryonic antigen as therapeutic vaccines against tumors

Saccharomyces cerevisiae engineered to express antigens specific to or associated with tumor cells like Ras gene mutation or carcinoembryonic antigen had been shown to act as a potential therapeutic vaccine. They had been shown to increase in Dendritic cells, Helper T cells $\left(\mathrm{CD}^{+}\right)$and Cytotoxic T cells (CD 8+), providing cell-mediated immunity. These vaccines prepare the immune system of the host only to act against the infected cells but also to not act against the normal cells. Effect of different doses, frequency of doses, number of sites of dose administration and side effects like autoimmune responses had been monitored on mouse models against the control groups. Recombinant Saccharomyces cerevisiae, along with popular anticancer drugs like Cisplatin and gemcitabine, can modulate the immune responses in a specific manner. Positive results against infected cells with these recombinant vaccines with no or negligible side effects have made them a potential treatment choice against conventional chemotherapy. Specific immune responses had been generated in carcinogen-induced lung tumors in mice when treated with specific Ras mutation expressing recombinant yeast in a dose-dependent manner enhanced by various booster doses (Fig-2). It had been shown $90 \%$ of 33 patients treated with Ras mutation expressing Saccharomyces cerevisiae generated Ras specific $\mathrm{T}$ cells with no toxicity. Recombinant Saccharomyces cerevisiae expressing carcinoembryonic antigen had increased the number of specific dendritic cells, $\mathrm{T}$ cells and co-stimulatory molecules in mice models. T helper cells response had been increased in mice upon an increasing number of sites of administration.

Tumor cells evade the immune system as they are not presented to $\mathrm{T}$ cells by Antigen Presenting Cells, and also, not strong co-stimulatory signals are produced. Recombinant Saccharomyces cerevisiae had been shown to activate dendritic cells, which process antigen and present it on Class I and Class II, eliciting cytotoxic cell and helper $\mathrm{T}$ cell responses. Also, they are being tested along with conventional drugs. Due to their less toxic effects in initial testing, they can be considered for other diseases as well. The only question which arises now is how to generalize these vaccines and make them cost-effective if they pass clinical trials.

\section{Therapeutic potential of Saccharomyces cerevisiae to treat neonatal diarrhea in piglets}

Neonatal diarrhea reduces the production potential and growth rates of pigs. This has increased dependence on medicines like antibiotics which in turn has led to drug resistance in pigs. To counter this problem, the effect of a single dose of Saccharomyces cerevisiae CNCM-1-1079 had been monitored in pigs suffering diarrhea for 7 days. A decrease in the number of diarrhea days $(\mathrm{p}=0.008)$ and the fecal score ( $\mathrm{p}=0.01$ ),i.e., making feces firmer, has been observed.

There had been a significant decrease in the number of diarrhea days in the first week in the experiment group ( $\mathrm{n}=23, \mathrm{p}=0.008$ ) as compared to the control group, which received a placebo $(\mathrm{n}=23)$ upon administration of Saccharomyces cerevisiae CNCM-11079. Both the groups had the same birth weight and parity and only supplement of Saccharomyces cerevisiae CNCM-1-1079 had affected days of diarrhea. More piglets had a lower fecal score during the first 7 days of treatment $(p<0.01)$. A lower fecal score indicates firm feces, and a higher fecal score 
indicates watery feces characteristic of diarrhea. A negative correlation between Average Daily Weight and diarrhea days had been found $(\mathrm{p}<0.01)$. A positive correlation between the number of days and mortality had been found $(\mathrm{p}<0.05)$.

Single-dose of Saccharomyces cerevisiae var boulardii (CNCM-1-1079) given after birth (within 24h) to preweaning piglets had decreased the number of diarrhea days, and a negative correlation had been established between average daily weight and diarrhea days. Thus, yeast had improved the average weight of the piglets, which is lost in diarrhea. Also, there had been a positive correlation between diarrhea and mortality. So, this yeast had decreased the chances of mortality indirectly. Saccharomyces boulardii reduces diarrhea by improving osmotic balance. It had been proven rats treated by Saccharomyces boulardii increased expression of sodium/glucose co-transporter 1 and helped in water, electrolyte and glucose reabsorption. For pig production and avoiding drug resistance and toxicity in them, supplementation of Saccharomyces boulardii is a good option as enteric health can affect the lifetime health of pigs.

\section{Training of the immune system by chitin content in the cell wall of Saccharomyces cerevisiae}

Gut microbiota protects against pathogens by not allowing them to grow and also by training the immune system to act against it in the future. Similarly, Saccharomyces cerevisiae had been proven to be training stimuli for monocytes. Saccharomyces cerevisiae had trained cells to effectively produce cytokines like IL-6 and TNF- $\alpha$. This immunomodulation is mediated by chitin which induces epigenetic modifications.

Primary stimulation of monocytes with Saccharomyces cerevisiae (experiment group) and Candida albicans (positive control group) and secondary stimulation with lipopolysaccharides, Candida albicans or Pam3Cys4 in the experimental group had increased TNF- $\alpha$ similar to the control group. Different amounts of increase in TNF- $\alpha$ had been observed on treatment with yeasts from different sources, the clinical strain being more potent. Trained immune response (TNF- $\alpha$, IL- 6 ) and cytokine production had been induced by chitin isolated from cell walls of different strains of Saccharomyces cerevisiae. Monocytes trained with chitin, i.e., given primary stimulus of chitin, had shown a high percentage of E. coli containing and Candida albicans containing phagolysosomes which can degrade the pathogen. Histone methyltransferase had been inhibited by chitin trained immunity suggesting epigenetic changes involved by the action of chitin.

Trained immunity is a type of defense against pathogens due to the ability to fight pathogens provided by an initial encounter with a training microbe. Primary stimulation with Saccharomyces cerevisiae strains trains the immune system for any second stimuli due to chitin content in the cell wall. Chitin trains monocytes by epigenetic modulations like methylation, and it had been shown that betaglucan induced trained immunity is due to epigenetic mechanisms. Although chitin content is considered favorable, there had been some cases of fungi increasing a disease like intestinal inflammation increases inflammation in colitis models. So, their proper effect and dose are to be tested.

\section{Therapeutic potential of recombinant yeast expressing} glucagon-like peptide -2 in the intestine of weaned rats

During the weaning period, enteric health is established and affects the long-term gut structure, functioning and microbiota. So, to improve gut health during the weaning period, therapeutic potentials of the recombinant yeast expressing glucagon-like peptide- 2 had been monitored by administrating these recombinant yeasts to the weaned rats for 14 days. It had been observed that recombinant yeasts had stimulated erythrocyte proliferation $(\mathrm{p}<0.01)$, had increased Average Daily Weight $(\mathrm{p}<0.05)$, had 
increased height of villus in the jejunum $(\mathrm{p}<0.05)$ and had increased activity of digestive enzymes in the jejunum $(\mathrm{p}<0.01)$.

Recombinant Glucagon-like peptide-2 (GLP-2) expressing Saccharomyces cerevisiae had significantly stimulated enterocyte proliferation $(\mathrm{p}<0.01)$ in vitrostudies as compared to the control and was similar to rats treated with human recombinant GLP-2 ( $>0.05)$. After 14 days of supplementation of $480 \mathrm{uL}$ of water, non-recombinant yeasts, GLP-2 Saccharomyces cerevisiae and human recombinant GLP-2 (rh-GLP2) to 4 groups of rats (each group $n=12$ ), it had been observed- Average Daily Gain of GLP yeast group had been higher than that in the water-treated control group and non-recombinant yeast group $(\mathrm{p}<0.01)$ and had been equivalent to the positive control group rhGLP2 ( $>0.05)$. Height of mucosal villus had significantly increased in GLP 2 Saccharomyces cerevisiae group, but there was no difference in crypt depth in all the groups. Crypt cells in the jejunum region had shown proliferation. There had been a significant increase in activities of digestive enzymes like Creatine Kinase (CK), Alkaline Phosphatase (AP) and Lactate Dehydrogenase (LDH) in the jejunum $(\mathrm{p}<0.01)$.

Glucagon-like peptide-2 (GLP-2) has been known to stimulate mucosal growth, absorption and enzyme secretion. The effect of recombinant yeast had been seen in the jejunum as the receptors for GLP-2 are present in the jejunum predominantly. Recombinant yeast had stimulated erythrocyte proliferation $(\mathrm{p}<0.01)$ as it reduced apoptosis by inhibition of proapoptotic caspases-3, caspase-7 and caspase-9. An increase in the activity of digestive enzymes had been observed $(\mathrm{p}<0.01)$. The previous study had shown that GLP-2 had increased villus height due to an increasing number of crypt cells upon administration of $40 \mathrm{ug} / \mathrm{kg} /$ day in pigs.

\section{Therapeutic effects of Saccharomyces cerevisiae and} Saccharomyces boulardii on intestinal dysmotility

Saccharomyces cerevisiae is known to have probiotic potentials, and this makes it a good candidate to prevent and treat gut-related problems. Stressinduced intestinal dysmotility suffering mice had been treated with Saccharomyces cerevisiae and genetically similar Saccharomyces boulardii. Mice had been sacrificed, and propagating contractile clusters velocity, frequency and amplitude had been monitored with the help of video recordings. It had been observed that Saccharomyces boulardii is quite effective as well as yeast supernatants were effective in opening new scopes to find bioactive compounds involved.

Propagating Contractile Clusters (PCC) velocity and frequency in jejunum had been decreased but had been increased in the colon upon induction of dysmotility in the mice. However, the amplitude of PCC had increased significantly in the jejunum $(\mathrm{p}<0.01)$. PCC velocity had been increased by $43 \%$ $(\mathrm{p}<0.01)$ and frequency had been increased by $69 \%$ $(\mathrm{p}<0.01)$ in the jejunum and PCC velocity had been decreased by $32 \%(<0.01)$, and frequency had been decreased by $29 \%(\mathrm{p}=0.01)$ in colon on treatment with Saccharomyces boulardii. Amplitude change had not been significant. PCC velocity had been increased by $14 \%$ ( $\mathrm{p}=0.013)$, and frequency had been increased by $74 \%(p<0.001)$ in the jejunum and PCC velocity had been decreased by $37 \%(\mathrm{p}<0.01)$ in the colon on treatment with Saccharomyces cerevisiae. But there had been an insignificant change in the frequency in the colon region $(\mathrm{p}=0.994)$. Amplitude had increased by $27 \%(p=0.013)$ in the jejunum. However, the amplitude increase had been insignificant in the colon. Supernatant of centrifuged Schharomyces boulardii increased velocity $(\mathrm{p}<0.01)$ and frequency $(\mathrm{p}=0.067)$ in the jejunum but decreased velocity $(\mathrm{p}=0.038)$ and frequency ( $p=0.024)$. There was no significant change in amplitude in both regions. The supernatant of centrifuged Saccharomyces cerevisiae only significantly decreased velocity in the colon $(\mathrm{p}=0.002)$, 
increased frequency in the jejunum $(\mathrm{p}=0.003)$ and decreased amplitude in the jejunum ( $\mathrm{p}=0.0016)$.

Saccharomyces boulardii and Saccharomyces cerevisiae both had shown the ability to reverse dysmotility. However, it had been observed Saccharomyces boulardii is more efficient. This can occur due to their different response-ability concerning temperature, acidic stressors and growth characteristics. Also, it had been observed supernatants have therapeutic potentials of the yeast cell as they were efficient in treating intestinal dysmotility. Thus, to understand this bioactive molecule in the supernatant need to be identified and also it would decrease the need for administration which sometimes may not be safe. The therapeutic action of these yeasts starts within 10 minutes, indicating that the therapeutic effect is drug-like.

\section{The therapeutic effect of Saccharomyces cerevisiae in irritable bowel syndrome}

Intestinal Bowel Syndrome is a common intestinal disorder that causes a lot of abdominal pain along with bloating. Two random clinical studies had shown an effect of Saccharomyces cerevisiae CNCM I- 385 on Intestinal Bowel Syndrome was analyzed. It was observed that yeast relieved symptoms in affected individuals during the second month, significantly reducing abdominal pain and bloating.

Abdominal discomfort had been significantly different between the experimental group ( IBS$\mathrm{C}>2$ ) and the placebo group ( $\mathrm{p}=0.0417)$, and there had been bloating trend in favor of the active group at the end of two month period. IBS $-\mathrm{C}>2$ is the group suffering from irritable bowel syndrome (IBS) constipation type (C) with abdominal discomfort less than or equal to the score of 2 . The maximum effect, however, had been seen in the second month. Bloating had been decreased in IBS-C ( $\mathrm{p}=0.0463)$, in IBS- $\mathrm{C}>2 \quad(\mathrm{p}=0.0145)$ and in intent to treat (ITT) $(\mathrm{p}=0.0105)$ populations. Abdominal pain also had reduced in all three populations. In the IBS-C group, abdominal pain $(\mathrm{p}<0.0001)$ as well as bloating $(\mathrm{p}<0.0001)$ had reduced significantly in both the active group and placebo group. IBS-C $>2$ had slightly higher abdominal pain than the IBS -C group but abdominal pain within active and placebo groups of IBS-C >2 reduced significantly. A significant improvement had been observed in stool consistency upon administration of the yeast.

From the meta-analysis of two random clinical trials, it became clear that Saccharomyces cerevisiae, to a large extent, decreased abdominal pain and bloating. However, the effect of yeast can be seen in the second month. This is because there is some delay before the analgesic effect of the yeast to relieve abdominal pain.

Clinical Trials : as extracted from clinicaltrials.gov

\begin{tabular}{|l|l|l|}
\hline Sl no. & Title of the Clinical Trials & NCT Number \\
\hline 1 & $\begin{array}{l}\text { A Comparison of Three } \\
\text { Medications to Treat } \\
\text { Diarrhea in Adults. }\end{array}$ & NCT00807326 \\
\hline 2 & $\begin{array}{l}\text { Impact of Probiotics in } \\
\text { Modulation of Intestinal } \\
\text { Microbiota }\end{array}$ & NCT01895530 \\
\hline 3 & $\begin{array}{l}\text { The Effect of Probiotics } \\
\text { on E. Coli-induced } \\
\text { Gastroenteritis } \\
\text { (PROTETEC) }\end{array}$ & NCT01709266 \\
\hline 4 & $\begin{array}{l}\text { Impact of Probiotics on } \\
\text { the Intestinal Microbiota }\end{array}$ & NCT01609660 \\
\hline 5 & $\begin{array}{l}\text { Effects of S. Boulardii and } \\
\text { Amoxicillin/Clavulanate } \\
\text { on Gut Microbiota }\end{array}$ & NCT01473368 \\
\hline 6 & $\begin{array}{l}\text { Effects of Pre-, Pro- \& } \\
\text { Anti-biotics on Gut } \\
\text { Microbiota }\end{array}$ & NCT01414010 \\
\hline
\end{tabular}

A comparison of three medications to treat diarrhea Combined treatment of Loperamide-simethicone 2 $\mathrm{mg} / 125 \mathrm{mg}$ caplets and chewable tablets along with probiotic Saccharomyces boulardii $250 \mathrm{mg}$ capsules were provided to the patients. This is a phase 4 
clinical trial. Loperamide-simethicone was found superior to Saccharomyces boulardii. All the medications proved that they prevented the recurrence of diarrhea and reduces bowel discomfort, Results published:

https://pubmed.ncbi.nlm.nih.gov/26541878/

Impact of Probiotics in the modulation of Intestinal Microbiota

Supplement of Saccharomyces boulardii (100mg yeast capsule daily for seven days prior to surgery ) was given to patients suffering from colorectal cancer and who was going to take resection colon. The expression of some specific genes ( IL10, IL1B, IL23A, TNF, IL12B, INFJ, IL17A) of intestinal cells was studied prior to surgery.

Results published: Not provided

The effect of Probiotics on E. Coli-induced Gastroenteritis (PROTETEC)

Selected candidates were provided with placebo and probiotics supplements for two weeks twice daily. Then they were subjected to enterotoxigenic E. Coli (ETEC). ETEC increased watery stool output and GIrelated problems. The probiotics were not effective in providing resistance against ETEC.

Results published:

https://pubmed.ncbi.nlm.nih.gov/25369827/

Impact of Probiotics on intestinal Microbiota

A phase 4 clinical trial was done on patients treated with colorectal cancer, where they were treated with $100 \mathrm{mg}$ of saccharomyces boulardii every day. An intestinal biopsy was done to study the efficacy of probiotics under stressful conditions.

Results published: Not provided

Effects of Saccharomyces boulardii and Amoxicillin/Clavulanate on Gut microbiota

The study was done to understand the effects of probiotic Saccharomyces boulardii(500mg, twice daily) and antibiotic drug Amoxicillin/Clavulanate(875/125 mg 2 times daily) on healthy individuals. This combined treatment resulted in the production of secondary bile acid resulting in the prevention of antibiotic-related $C$. difficile infection.
Results published:

https:/pubmed.ncbi.nlm.nih.gov/30881353/

Effects of pre-, pro-, and antibiotics on Gut Microbiota

The study was done to understand the reversible effects of prebiotic (Trametes versicolor, 1200mg thrice daily), probiotic (Saccharomyces boulardii, 250mg thrice daily) and antibiotic (Amoxicillin, 250mg thrice daily) agent on a healthy intestinal microbiota. It is believed that the treatment induces a reproducible fecal microbiota that is reversible once the treatment is discontinued.

Results published: Not provided

\subsection{Saccharomyces boulardii}

Effects on the mucosal system due to oral administration of Saccharomyces boulardii

This article is regarding the experiments performed on a group of rats and humans who have been administered with oral doses of either lyophilized or heat-killed Saccharomyces boulardii. There were no gastric complications in the species. The oral administration of the yeast was given for a period of five days. The intestine was removed in the rats, and in the case of humans, a biopsy on the intestine was performed. Enzymatic activity was studied in vitro. The incorporation of Saccharomyces boulardii into the enzymatic activity was also studied by enzyme assays.

Human studies: There was no change in villus height or crypt depth. But an increased activity of maltase, lactase and sucrase was observed. The overall mucosal protein content was not changed. The volunteers did not suffer through any abdominal complications during the experiment tenure.

Animal studies: The results were similar to that of Human studies. Yeast cells were found on the inner lumen of the intestine in contact with the villus cells, and there were no signs of inflammation. The overall 
protein content was unchanged, but there was a slight increase in aminopeptidase activity.

Saccharomyces boulardii induces no toxic effects on the gut. It is given as a treatment for diarrhea and for other diseases that include degradation of the intestinal microbiota. Saccharomyces boulardii is antibiotic-resistant and protects intestinal cells from gastric activity. Human fecal matter is rich in yeast cells, whereas in rats, the villus cells do incorporate with the yeast cells, and there is a less amount of excretion. The lactase biogenesis levels were similar both in control mice and treated mice. The mannose content in the cell wall may be the reason behind the increased disaccharide activity. The rate of DNA synthesis was studied to justify the increased disaccharide activity by incorporating thymidine. No difference was observed.

\section{Saccharomyces boulardii in the prevention of relapse of Crohn's Disease and as a supporting treatment}

In several studies, it has been proved that 5aminosalicylic acid (5-ASA, mesalamine) is a more effective treatment than placebo. In this study, the authors performed an experiment with 32 patients placed in two groups, A and B. They were all diagnosed with Crohn's disease of the ileum and colon. Group A patients were given mesalazine 500 $\mathrm{mg}$ in a sustained-release preparation in ethylcellulose microgranules (Pentasa), 2 capsules three times a day. Group B has given Saccharomyces Boulardii $500 \mathrm{Mg} 2$ capsules in the morning 1 Pentasa $500 \mathrm{mg}$ two capsules twice a day. It was found that clinical relapse was found more in Group A patients compared to Group B. The authors tried to explain the reason behind this result.

The remission of the disorder was measured in terms of Crohn's disease activity index (CDAI). CDAI $<150$ was considered safe. In Group A, six patients suffered clinical relapse within the time frame of 6 months, while in Group B, only one patient suffered from clinical remission.
It was found that patients treated with mesalamine and Saccharomyces boulardii (6.25\%) had a lesser chance of clinical remission compared to that of patients treated with mesalamine (37.5\%) alone. Crohn's disease is believed to destroy the intestinal microbiota, and regeneration takes a long time, during which chemical relapses are very common. Saccharomyces boulardii is very similar to Saccharomyces cerevisiae due to which on the administration of this strain, the same kind of antibodies is produced in the host system, which is very specific against Crohn's disease. Other than this, Saccharomyces boulardii is a probiotic agent that exerts certain trophic effects on the intestine, for example, regulation of immune response by the release of $\operatorname{IgA}$, reducing the effects of antibioticinduced diarrhea, and regeneration of the intestinal microbiota.

Thus, Saccharomyces boulardii has shown positive results in improving the CDAI parameters. Saccharomyces boulardii is yet to undergo several trials before being accepted for routine use as maintenance treatment for Crohn's disease.

\section{Saccharomyces boulardii in the enhancement of immune response in host organism in case of Clostridium difficile}

The main area of interest in this study is to understand the action of the host immune response in the presence of a probiotic agent, Saccharomyces boulardii, when affected by Clostridium difficile. The pathogen produces two toxins, $\mathrm{A}$ and $\mathrm{B}$. Toxin $\mathrm{A}$ is primarily responsible for the infection (fluid secretion, inflammation). Toxin B causes injury to the colon. Saccharomyces boulardii is a probiotic agent that reduces the effects of diarrhea and clinical remission. Other than the antibodies produced by the host cells in response to toxin A, Saccharomyces boulardii increased the secretion of immunoglobulin A. Four groups of $B A L B / c$ mice were taken for the study. The first group was for control, the second 
group was treated with formalin-inactivated toxoid A, the third group was treated with Saccharomyces boulardii with drinking water, and the fourth group was treated with both Saccharomyces boulardii and inactivated toxoid A. After a certain period, they were all sacrificed, and their intestine and serum samples were taken for further studies. Antibodies against the toxins were studied by ELISA.

IgA levels were increased in group 3 by 1.9 fold compared to that of controls. In the fourth group, the increase was 1.8 fold, but the difference was that the IgA was toxin A specific. There was an increase of antitoxin A IgA when the rats were treated with both toxoid and Saccharomyces boulardii.

Coadministration of Saccharomyces boulardii with Clostridium difficile has resulted in the production of anti-A toxin IgA. Clostridium difficile has been studied, and it has been proved that they do not trigger a vigorous immune response; instead, they remain asymptomatic. Thus this study proves that Saccharomyces boulardii exhibit protective effects against diarrhea caused due to Clostridium difficile.

\section{Saccharomyces boulardii: A preventive treatment against bacterial translocation}

The case study portrays the effects of Saccharomyces boulardii on preventing bacterial translocation in rats affected by obstructive jaundice. Bacterial translocation is usually caused due to bacterial overgrowth in the intestine, deficiencies in the host immune system or due to increased permeability in the mucus layer or the epithelial cells.

The study was done with 60 adult rats taken into five different groups. Group 1 was the sham-operated group (they had mobilization of the common bile duct); Group 2 was the common bile duct ligation group; Group 3 was the Saccharomyces boulardii group; Group 4 was the ampicillin-sulbactam group, and group 5 was the Saccharomyces boulardii plus ampicillin-sulbactam group. All the rats were given a dose of saccharomyces boulardii along with antibiotics for 7 days. After this time period, the rat had laparotomy and samples of blood, liver, spleen and lymph nodes were taken for microbial studies. Bacterial translocation percentages were $0 \%$ in the sham-operated group, $83 \%$ in the first group, $42 \%$ in groups 3 and 4 and $33 \%$ in group 5. Bacterial translocation rates were $0 \%$ in the sham-operated group, $83 \%$ in group 2, $42 \%$ in group 3, $42 \%$ in group 4 and $33 \%$ in group 5 . In groups 2, 3, 4 and 5, the serum bilirubin levels, Alkaline phosphatase and alanine aminotransferase, and aspartate aminotransferase activities were significantly higher compared to Group 1. In groups 2, 3, 4 and 5, there is a decrease in the heights of ileal villi compared to that of group 1. Bacterial translocation can be caused due to several factors other than obstructive jaundice. This can lead to loss of the intestinal microbiota as well as the loss of occludin proteins that maintain the tight junctions of the epithelial cells of the intestine. Bacterial translocation in immunosuppressed patients may turn into fatal sepsis. Experimentally, Saccharomyces boulardii has been found very effective against bacterial translocation and improvement of intestinal barrier function.

\section{The effects of Saccharomyces boulardii CNCM I-745 in restoring intestinal microbiota after dysbiosis}

The review article is about understanding the intestinal microbial population in a healthy system as well as the population imbalance during dysbiosis. During a healthy environment, the intestinal microbiota is protected by Saccharomyces boulardii. Due to dysbiosis caused by diarrhea, there is a collapse of the original microbial species in the intestine. Saccharomyces boulardii also induce regeneration of the microbiota. Within the intestine, there is a large microbial population that produces several secondary metabolites (vitamin) and substances like butyrate that carry out several regulatory functions. The intestinal microbiota is also responsible for the maintenance of water and 
electrolyte levels and inducing a general immune response. On the other hand, in the case of dysbiosis, a disruption of the mucus layer causes inflammation, and the administration of antibiotics leads to the depletion of the microbial population that is present in the intestine. Food remains in the intestinal lumen for a long along with osmotic water resulting in the formation of watery stools. The immune response that is induced by the bacteria increases the leukocyte infiltration and damages the potential bacterial population helpful for the intestine. Saccharomyces boulardii is resistant to antibiotics and can withstand oxidative stress. They do not transfer their resistant genes to other bacteria due to their fungal nature. They are not predominantly found in the intestinal microbiota and excreted within 3 days of administration. It also exhibits high acid tolerance, which is optimum for survival in the intestine. The beta glycans present in the fungal cell wall bind with the receptors on the dendritic cells, thus inducing an immune response. The NF- $\mathrm{BB}$ and the MAP kinase pathways are inhibited that demonstrates the antiinflammatory activity. The cell wall provides a substrate for increased microbial activity, and there is an increased SCFA Production.

This study tells us the importance of Saccharomyces boulardii in the regeneration of intestinal microbiota after the treatment of an antibiotic. Diarrhea is a common factor of death for many immunodeficient patients and even among children and old people. A steady administration of Saccharomyces boulardii can successfully maintain the gut flora and reduce the risk of diarrhea. Other than this, it also provides several positive effects like reducing weight by the production of butyrate and relieves constipation by the secretion of anti-inflammatory chemokines.

\section{The adaptation capability of saccharomyces boulardii} in host organisms makes it a perfect probiotic agent.

The article is about the genetic makeup of Saccharomyces boulardii, which makes it different from other yeast species and a successful probiotic agent. Saccharomyces boulardii compete with pathogens in the gut and can even withstand the high $\mathrm{pH}$ in the intestine. The qualities of toxicity and antibiotic resistance are not shared with any other bacteria. In this article, the author tried to explain the reason behind the distinguishable characters of Saccharomyces boulardii and its mode of mechanism. Genes that were expressed for protein synthesis (RPL31A, RPL41A, RPS24B, RPL2B and RSA3) and stress response (HSP26, SSA3, SED1, HSP42, HSP78 and PBS2) had a higher copy number. There was an increased number of alterations seen in SDH1 and WHI2 genes as compared to Saccharomyces cerevisiae, and this results in an increased amount of acetic acid production. The ability of faster pseudohyphal growth is due to increased expression of CDC42, DFG16, RGS2, CYR1 and CDC25. A point mutation in the PGM2 gene inhibits Saccharomyces boulardii from using galactose as a carbon source. The mechanism of yeast action can be divided into four groups: modulating an immune response, removal of the pathogen, antimicrobial properties and certain trophic effects. Removal of the pathogen is achieved by the binding of the pathogen with the yeast and further hindering their pathway of virulence. Saccharomyces boulardii release several metabolites (bacteriocins, hydrogen peroxide) and polyamines that inhibit the growth of the pathogens. The intestine provides a favorable environment for the growth of Saccharomyces boulardii, and thus it actively competes with the microbes for nutrition. Saccharomyces boulardii exhibits certain antimicrobial properties. A number of proteins (54$\mathrm{kDa}, 63-\mathrm{kDa}$ and $120-\mathrm{kDa}$ proteins) synthesized by the yeast has a number of antimicrobial effects. The genomics of Saccharomyces boulardii explained the reason for its adaptability in the human gut. The immunomodulatory response that is triggered by the yeast species and the suppression of IL-8 has a unique two-fold effect on the intestinal microbiota. There are several other questions that remained unanswered 
about the $54-\mathrm{kDa}, 63-\mathrm{kDa}$ and $120-\mathrm{kDa}$ proteins that are secreted by the yeast and have antimicrobial activity. Their specific mechanism of cleaving the microbial toxin is not fully understood. The mechanism of regulation of the immune response by Saccharomyces boulardii is not defined completely.

Clinical evidence: as extracted from clinicaltrials.gov

\begin{tabular}{|c|c|c|}
\hline $\begin{array}{l}\text { Sl } \\
\text { no }\end{array}$ & Title of the Clinical Trials & NCT Number \\
\hline 1 & $\begin{array}{l}\text { A Comparison of Three } \\
\text { Medications to Treat Diarrhea } \\
\text { in Adults. }\end{array}$ & NCT00807326 \\
\hline 2 & $\begin{array}{l}\text { Impact of Probiotics in } \\
\text { Modulation of Intestinal } \\
\text { Microbiota }\end{array}$ & NCT01895530 \\
\hline 3 & $\begin{array}{l}\text { The Effect of Probiotics on E. } \\
\text { Coli-induced Gastroenteritis } \\
\text { (PROTETEC) }\end{array}$ & NCT01709266 \\
\hline 4 & $\begin{array}{l}\text { Impact of Probiotics on the } \\
\text { Intestinal Microbiota }\end{array}$ & NCT01609660 \\
\hline 5 & $\begin{array}{l}\text { Effects of } S . \text { Boulardii and } \\
\text { Amoxicillin/Clavulanate on } \\
\text { Gut Microbiota }\end{array}$ & NCT01473368 \\
\hline 6 & $\begin{array}{l}\text { Effects of Pre-, Pro- \& Anti- } \\
\text { biotics on Gut Microbiota }\end{array}$ & NCT01414010 \\
\hline
\end{tabular}

\section{A comparison of three medications to treat diarrhea}

Combined treatment of Loperamide-simethicone 2 $\mathrm{mg} / 125 \mathrm{mg}$ caplets and chewable tablets along with probiotic Saccharomyces boulardii $250 \mathrm{mg}$ capsules were provided to the patients. This is a phase 4 clinical trial. Loperamide-simethicone was found superior to Saccharomyces boulardii. All the medications proved that they prevented the recurrence of diarrhea and reduces bowel discomfort, Results published:

https://pubmed.ncbi.nlm.nih.gov/26541878/

Impact of Probiotics in the modulation of Intestinal Microbiota

Supplement of Saccharomyces boulardii (100mg yeast capsule daily for seven days prior to surgery ) was given to patients suffering from colorectal cancer and who was going to take resection colon. The expression of some specific genes ( IL10, IL1B, IL23A, TNF, IL12B, INFJ, IL17A) of intestinal cells was studied prior to surgery.

Results published: Not provided

The effect of Probiotics on $E$. coli-induced Gastroenteritis (PROTETEC)

Selected candidates were provided with placebo and probiotics supplements for two weeks twice daily. Then they were subjected to enterotoxigenic E. coli (ETEC). ETEC increased watery stool output and GIrelated problems. The probiotics were not effective in providing resistance against ETEC.

Results published:

https://pubmed.ncbi.nlm.nih.gov/25369827/

Impact of Probiotics on intestinal Microbiota

A phase 4 clinical trial was done on patients treated with colorectal cancer, where they were treated with $100 \mathrm{mg}$ of Saccharomyces boulardii every day. An intestinal biopsy was done to study the efficacy of probiotics under stressful conditions.

Results published: Not provided

Effects of Saccharomyces boulardii and Amoxicillin/Clavulanate on Gut microbiota

The study was done to understand the effects of probiotic Saccharomyces boulardii $(500 \mathrm{mg}$, twice daily) and antibiotic drug Amoxicillin/ Clavulanate (875/ $125 \mathrm{mg} 2$ times daily) on healthy individuals. This combined treatment resulted in the production of secondary bile acid resulting in the prevention of antibiotic-related $C$. difficile infection.

Results published:

https://pubmed.ncbi.nlm.nih.gov/30881353/

Effects of pre-, pro-, and antibiotics on Gut Microbiota

The study was done to understand the reversible effects of prebiotic (Trametes versicolor, 1200mg thrice daily), probiotic (Saccharomyces boulardii, 250mg thrice daily) and antibiotic (Amoxicillin, 250mg thrice daily) agent on a healthy intestinal microbiota. It is believed that the treatment induces a 
reproducible fecal microbiota that is reversible once the treatment is discontinued.

Results published: Not provided

\section{IV.CONCLUSION}

This research review's purpose is to help the reader understand different aspects posed by the research on therapeutic applications of yeast 'Saccharomyces'. This is significant because it gives insights into how Saccharomyces can be used as a potential probiotic/ vaccine/ immunomodulator in various diseases. There has been much research and discussion of these opinions of Saccharomyces cerevisiae and Saccharomyces boulardii and their therapeutic application. Most of the research found was on the possible treatment of various diseases by different pathways. In some cases, such as Intestinal disorders, they act as probiotics. Whereas sometimes they show potential as a recombinant vaccine. Other times it is used to elicit an immune response and secrete IgA. They also found applications as antioxidants and anticancer agents. More research and testing are required to gain a better understanding of the therapeutic applications of yeast 'Saccharomyces'.

\section{ACKNOWLEDGEMENT}

We would like to thank our supervisor/guide Bharat Kwatra, from Invenzion Labs Inc., whose expertise was invaluable in formulating the research questions, methodology and drawing conclusions. His insightful feedback and guidance pushed us to sharpen our thinking and brought our work to a higher level.

\section{HUMAN AND ANIMAL RIGHTS}

No Animals/Humans were used for studies that are base on this research.

\section{AVAILABILITY OF DATA AND MATERIALS}

The author confirms that the data supporting the findings of this research are available within the article.

\section{FUNDING ACKNOWLEDGEMENT CONFLICT OF INTEREST}

AND

The authors whose names are listed immediately above certify that they have NO affiliations with or involvement in any organization or entity with any financial interest (such as honoraria; educational grants; participation in speakers' bureaus; membership, employment, consultancies, stock ownership, or other equity interest; and expert testimony or patent-licensing arrangements), or nonfinancial interest (such as personal or professional relationships, affiliations, knowledge or beliefs) in the subject matter or materials discussed in this manuscript.

\section{REFERENCES}

[1]. Buts, J. P., Bernasconi, P., Van Craynest, M. P., Maldague, P. \& Meyer, R. Response of human and rat small intestinal mucosa to oral administration of Saccharomyces boulardii. Pediatr. Res. 20, (1986).

[2]. Guslandi, M., Mezzi, G., Sorghi, M. \& Testoni, P. A. Saccharomyces boulardii in maintenance treatment of Crohn's disease. Dig. Dis. Sci. 45, (2000).

[3]. Qamar, A. et al. Saccharomyces boulardii stimulates intestinal immunoglobulin a immune response to Clostridium difficile toxin $\mathrm{A}$ in mice. Infect. Immun. 69, (2001).

[4]. Czerucka, D. \& Rampal, P. Experimental effects of Saccharomyces boulardii on diarrheal pathogens. Microbes and Infection vol. 4 (2002).

[5]. Blanquet, S., Meunier, J. P., Minekus, M., MarolBonnin, S. \& Alric, M. Recombinant Saccharomyces cerevisiae expressing $\mathrm{P} 450$ in 
artificial digestive systems: A model for biodetoxication in the human digestive environment. Appl. Environ. Microbiol. 69, (2003).

[6]. Mansour-Ghanaei, F., Dehbashi, N., Yazdanparast, K. \& Shafaghi, A. Efficacy of Saccharomyces boulardii with antibiotics in acute amoebiasis. World J. Gastroenterol. 9, (2003).

[7]. Geyik, M. F. et al. The effects of Saccharomyces boulardii on bacterial translocation in rats with obstructive jaundice. Ann. R. Coll. Surg. Engl. 88, (2006).

[8]. Garrait, G., Jarrige, J. F., Blanquet, S., Beyssac, E. \& Alric, M. Recombinant Saccharomyces cerevisiae strain expressing a model cytochrome P450 in the rat digestive environment: Viability and bioconversion activity. Appl. Environ. Microbiol. 73, (2007).

[9]. Şahin, T. et al. Effects of the probiotic agent Saccharomyces boulardii on the DNA damage in acute necrotizing pancreatitis induced rats. Hum. Exp. Toxicol. 26, (2007).

[10]. Ardiani, A., Higgins, J. P. \& Hodge, J. W. Vaccines based on whole recombinant Saccharomyces cerevisiae cells. FEMS Yeast Research vol. 10 (2010).

[11]. Matuo, R. et al. Saccharomyces cerevisiae as a model system to study the response to anticancer agents. Cancer Chemotherapy and Pharmacology vol. 70 (2012).

[12]. Kelesidis, T. \& Pothoulakis, C. Efficacy and safety of the probiotic Saccharomyces boulardii for the prevention and therapy of gastrointestinal disorders. Therapeutic Advances in Gastroenterology vol. 5 (2012).

[13]. Feizizadeh, S., Salehi-Abargouei, A. \& Akbari, V. Efficacy and safety of Saccharomyces boulardii for acute diarrhea. Pediatrics vol. 134 (2014).

[14]. Hancox, L. R. et al. Effect of a single dose of Saccharomyces cerevisiae var. boulardii on the occurrence of porcine neonatal diarrhoea. Animal 9, (2015).

[15]. Moré, M. I. \& Swidsinski, A. Saccharomyces boulardii CNCM I-745 supports regeneration of the intestinal microbiota after diarrheic dysbiosis - A review. Clinical and Experimental Gastroenterology vol. 8 (2015).

[16]. Rizzetto, L. et al. Fungal chitin induces trained immunity in human monocytes during crosstalk of the host with Saccharomyces cerevisiae. J. Biol. Chem. 291, (2016).

[17]. West, C., Stanisz, A. M., Wong, A. \& Kunze, W. A. Effects of Saccharomyces cerevisiae or boulardii yeasts on acute stress induced intestinal dysmotility. World J. Gastroenterol. 22, (2016).

[18]. Zhang, Z., Wu, X., Cao, L., Zhong, Z. \& Zhou, Y. Generation of glucagon-like peptide-2expressing Saccharomyces cerevisiae and its improvement of the intestinal health of weaned rats. Microb. Biotechnol. 9, (2016).

[19]. Hudson, L. E. et al. Characterization of the probiotic yeast Saccharomyces boulardii in the healthy mucosal immune system. PLoS One 11, (2016).

[20]. Kwatra, B. Tinospora Crispa As A Future Cure For Obesity/Cholesterol. Int. J. Sci. Technol. Res. 6, 340-341 (2017).

[21]. Kwatra, B. HACKING THE BLOOD-BRAIN BARRIER. Eur. J. Biol. Med. Sci. Res. 5, 10-13 (2017).

[22]. Kwatra, B. LOCATOR THEORY FOR ELEMENTS IN PERIODIC TABLE 'LEPT'. Glob. J. Pure Appl. Chem. Res. 5, 9-10 (2017).

[23]. Cayzeele-Decherf, A. et al. Saccharomyces cerevisiae CNCM I-3856 in irritable bowel syndrome: An individual subject meta-analysis. World J. Gastroenterol. 23, (2017).

[24]. Srinivas, B. et al. Evaluating the probiotic and therapeutic potentials of Saccharomyces cerevisiae strain (OBS2) isolated from 
fermented nectar of toddy palm. AMB Express 7, (2017).

[25]. Fakruddin, M., Hossain, M. N. \& Ahmed, M. M. Antimicrobial and antioxidant activities of Saccharomyces cerevisiae IFST062013, a potential probiotic. BMC Complement. Altern. Med. 17, (2017).

[26]. Appel-da-Silva, M. C., Narvaez, G. A., Perez, L. R. R., Drehmer, L. \& Lewgoy, J. Saccharomyces cerevisiae var. boulardii fungemia following probiotic treatment. Med. Mycol. Case Rep. 18, (2017).

[27]. Yu, L. et al. Saccharomyces boulardii Administration Changes Gut Microbiota and Attenuates D-Galactosamine-Induced Liver Injury. Sci. Rep. 7, (2017).

[28]. Duman-Scheel, M. Saccharomyces cerevisiae (Baker's Yeast) as an Interfering RNA Expression and Delivery System. Curr. Drug Targets 20, (2018).

[29]. Nandy, S. K. \& Srivastava, R. K. A review on sustainable yeast biotechnological processes and applications. Microbiological Research vol. 207 (2018).

[30]. Kwatra, B. Bioactive-Compounds: alternative to control Candida spp. Int. J. Sci. Res. Rev. 8, 221-223 (2019).

[31]. Kwatra, B. HYDROQUINONE: A novel growth inhibitor and apoptosis inducer in U-251 MG CELLS. Int. J. Med. Biomed. Stud. 3, 15-16 (2019).

[32]. Kwatra, B. Allicin-An After Digestion Antimicrobial Agent. ACTA Sci. Microbiol. 2, 48-51 (2019).

[33]. Kwatra, B. Effects of Mineral Separation by Time and Enteric Coating Mechanism for Calcium and Iron Absorption in Mammalia. Int. J. Sci. Res. 8, 1265-1270 (2019).

[34]. Kwatra, B. MECHANISMS OF PATTERN FORMATION OF FBP17 IN MAST CELLS. Int. J. Adv. Res. 7, 413-414 (2019).
[35]. Kwatra, B. Procuring Natural Dye for Solar Cell Using Leaf Waste. Int. J. Sci. Eng. Res. 7, 46-47 (2019).

[36]. Kwatra, B. EXPRESSION AND CHARACTERIZATION IN PICHIA PASTORIS BY CLONING OF DELTA 4 DESATURASE FROM ISOCHRYSIS GALBANA. Indian J. Appl. Res. 9, 1-2 (2019).

[37]. Kwatra, B. Holothuroidea (Sea Cucumber): Key to Anti-Aging. Int. J. Sci. Res. 8, 884 (2019).

[38]. Kwatra, B. A REVIEW ON POTENTIAL PROPERTIES AND THERAPEUTIC APPLICATIONS OF BROMELAIN. www.wjpps.com 8, 488-500 (2019).

[39]. Kwatra, B. A Review on Potential Properties and Therapeutic Applications of DHA and EPA. ijppr.humanjournals 16, 140-176 (2019).

[40]. Kwatra, B. THE SIMVASTATIN AND DMXAA ON THE CO-CULTURE OF B16.F10 MELANOMA CELLS AND MACROPHAGES SHOWS ANTITUMOR ACTIVITY. World J. Pharm. Res. 8, 1318-1319 (2019).

[41]. Czerucka, D. \& Rampal, P. Diversity of Saccharomyces boulardii CNCM I-745 mechanisms of action against intestinal infections. World J. Gastroenterol. 25, (2019).

[42]. Kumar, R. \& Kumar, P. Yeast-based vaccines: New perspective in vaccine development and application. FEMS Yeast Research vol. 19 (2019).

[43]. Kwatra, B. A REVIEW ON POTENTIAL PROPERTIES AND THERAPEUTIC APPLICATIONS OF LYCOPENE. Int. J. Med. Biomed. Stud. 4, 33-44 (2020).

[44]. Kwatra, B. Candidate genes of OCD interacts with human retrovirus to form new link in inflammatory hypothesis. Int. J. Sci. Appl. Res. 7, 1-2 (2020).

[45]. Kwatra, B. Maprovit 3, 6, 9: Perfect Companion of your Immune System to Fight Corona Virus Hit. Int. J. Sci. Res. 9, 241 (2020). 
[46]. Kwatra, B. A Review on Potential Properties and Therapeutic Applications of Vitamin D. Int. J. Sci. Res. 9, 682-691 (2020).

[47]. Kwatra, B. COLLAGEN SUPPLEMENTATION : THERAPY FOR SKIN DISORDERS: A REVIEW. World J. Pharm. Res. 9, 2504-2518 (2020).

[48]. Kwatra, B. A REVIEW ON POTENTIAL PROPERTIES AND THERAPEUTIC APPLICATIONS OF BRANCHED CHAIN AMINO. WORLD J. Pharm. Pharm. Sci. 9, 561588 (2020).

[49]. Kwatra, B. COLLAGEN SUPPLEMENTATION : THERAPY FOR THE PREVENTION AND TREATMENT OF OSTEOPOROSIS AND OSTEOARTHRITIS: A REVIEW. WORLD J. Pharm. Pharm. Sci. 9, 589-604 (2020).

[50]. Kwatra, B. A REVIEW ON POTENTIAL PROPERTIES AND THERAPEUTIC APPLICATIONS OF GRAPE SEED EXTRACT. World J. Pharm. Res. 9, 2519-2540 (2020).

[51]. Kwatra, B. Studies on People Employed in High Risk Workplace: Between Genetic Polymorphism for Tumor Necrosis Factor ( TNF- A ) and Blood Pressure. Int. J. Innov. Res. Technol. 6, 268-270 (2020).

[52]. Decherf, A. et al. Recovery of Saccharomyces cerevisiae CNCM i-3856 in vaginal samples of healthy women after oral administration. Nutrients 12, (2020).

[53]. de Campos Fraga-Silva, T. F. et al. Selenization of $S$. cerevisiae increases its protective potential in experimental autoimmune encephalomyelitis by triggering an intestinal immunomodulatory loop. Sci. Rep. 10, (2020).

[54]. Del Rivero, J. et al. A Case Report of Sequential Use of a Yeast-CEA Therapeutic Cancer Vaccine and Anti-PD-L1 Inhibitor in Metastatic Medullary Thyroid Cancer. Front. Endocrinol. (Lausanne). 11, (2020).

[55]. Wang, B. et al. Saccharomyces boulardii attenuates inflammatory response induced by
Clostridium perfringens via TLR4/TLR15MyD8 pathway in HD11 avian macrophages. Poult. Sci. 99, (2020).

[56]. Kaźmierczak-Siedlecka, K., Ruszkowski, J., Fic, M., Folwarski, M. \& Makarewicz, W. Saccharomyces boulardii CNCM I-745: A Nonbacterial Microorganism Used as Probiotic Agent in Supporting Treatment of Selected Diseases. Current Microbiology vol. 77 (2020).

[57]. Pais, P., Almeida, V., Yllmaz, M. \& Teixeira, M. C. Saccharomyces boulardii: What makes it tick as successful probiotic? Journal of Fungi vol. 6 (2020).

[58]. Gaziano, R., Sabbatini, S., Roselletti, E., Perito, S. \& Monari, C. Saccharomyces cerevisiae Based Probiotics as Novel Antimicrobial Agents to Prevent and Treat Vaginal Infections. Frontiers in Microbiology vol. 11 (2020).

[59]. Constante, M. et al. Saccharomyces boulardii CNCM I-745 modulates the microbiota-gutbrain axis in a humanized mouse model of Irritable Bowel Syndrome. Neurogastroenterol. Motil. (2020) doi:10.1111/nmo.13985.

[60]. Ribeiro, M. R. S. et al. Saccharomyces boulardii as therapeutic alternative in experimental giardiasis. J. Appl. Microbiol. (2020) doi:10.1111/jam.14941.

\section{Cite this article as :}

Olivia Mondal, Devanshi Khanna, Shalja Panwar, Shilpa Negi, Sneha Basu, "Systematic Review on Therapeutic Applications of Yeast 'Saccharomyces'", International Journal of Scientific Research in Science and Technology (IJSRST), Online ISSN : 2395-602X, Print ISSN : 2395-6011, Volume 8 Issue 2, pp. 174-197, March-April 2021. Available at doi : https://doi.org/10.32628/IJSRST218226 Journal URL : https://ijsrst.com/IJSRST218226 